Published in Applied Geochemistry, v. 14, pp. 423-432 (1999). () Elsevier Science Bv.

Geochemistry of natural and anthropogenic fall-out (aerosol and precipitation) collected in N W Mediterranean: two different multivariate statistical approaches.

Molinaroli E., Pistolato M. and Rampazzo G.

Dipartimento di Scienze Ambientali, Università di Venezia, Dorsoduro 2137, 30123

Venezia, Italy

Guerzoni S.

CNR, Istituto di Geologia Marina, via Gobetti 101, 40129 Bologna, Italy

Author for Correspondence:

Emanuela Molinaroli

Dipartimento di Scienze Ambientali, Università di Venezia, Dorsoduro 2137, 30123

Venezia, Italy 


\begin{abstract}
The chemical characteristics of the mineral fractions of aerosol and precipitation collected in Sardinia (NW Mediterranean) are highlighted by means of two multivariate statistical approaches. Two different combinations of classification and statistical methods for geochemical data are presented. It is shown that the application of cluster analysis subsequent to Q-Factor analysis better distinguishes among Saharan dust, Background pollution (Europe-Mediterranean) and Local aerosol from various source regions (Sardinia). Conversely, the application of simple cluster analysis was able to distinguish only between aerosols and precipitation particles, without assigning the sources (local or distant) to the aerosol. This method also highlighted the fact that crust-enriched precipitation is similar to desert-derived aerosol.

Major elements $(\mathrm{Al}, \mathrm{Na})$ and trace metal $(\mathrm{Pb})$ turn out to be the most discriminating elements of the analysed data set.

Independent use of mineralogical, granulometric and meteorological data confirmed the results derived from the statistical methods employed.
\end{abstract}

Keywords: multivariate statistical analysis, aerosol, precipitation, geochemistry Mediterranean. 


\section{INTRODUCTION}

Statistical analysis in atmospheric studies is a well-known and well-developed field (Morandi et al., 1987; Hopke, 1988; Van Malderen et al., 1992). This study presents an example of the use of cluster analysis (CA), Q-Factor analysis (Q-FA) and discriminant analysis (DA) in atmospheric geochemistry, in an attempt to classify mixed aerosol and precipitation samples on the basis of their major element and trace metal chemistry.

Atmospheric inputs to the Mediterranean are of particular interest, in view of the different areas of aerosol production on the north and south belts.

The principal source of natural "crustal" material in the Mediterranean is the Sahara, while industrialized and semi-industrialized Northern Europe is a source of anthropogenic "background" material to the atmosphere. These inputs have an important influence on both the mineralogical and chemical composition of the Mediterranean aerosol. Previous works have shown that the bulk aerosol in the lower troposphere in this area has two main components: sea salt aerosol and mineral aerosol (Bergametti et al., 1989; Correggiari et al., 1989; Chester et al., 1993a).

These aerosols are removed from the atmosphere to the sea surface by a combination of 'dry' (i.e., not involving aqueous phase) and 'wet' (precipitation scavenging) depositional modes, and these modes are geochemically different with respect to the solutions with which the aerosols come into contact. Estimates of dust transport and deposition in the Mediterranean have been made by the Group of Experts on the Scientific Aspects of Marine Pollution (GESAMP, 1989) using a model. The temporal variability of dust deposition rates may vary greatly because of the strong seasonal cycle in dust concentrations and the large seasonal variability of precipitation rates (Prospero, 1996).

The present study is based on a collection of six-month aerosol samples and one-year precipitation samples at a remote site. An overall sampling program was applied to investigate the geochemistry of natural (Saharan dust) and anthropogenic (Background) aerosols and to separate local versus distant inputs to the Western Mediterranean (Guerzoni et al., 1997). If and how crust-enriched precipitation is similar to desert-derived aerosol was also verified.

Two different ways of treating the data sets were planned, as follows:

1. a rank transformation was applied to the data and subsequently $\mathrm{CA}$ on the ranked data, followed by DA on the CA groups.

2. the data matrix was first reduced on the basis of Q-FA on the ranked data; then CA was applied to samples, with factor loading as new variables, and then DA on the CA groups.

The aims of this paper were to present the geochemical composition of insoluble aerosol and suspended particulate in precipitation and to show two different multivariate statistical approaches to the data sets; and then to translate statistical results into meaningful geological statements. The contributions of local versus distant sources were particularly highlighted.

\section{SAMPLING}

Chemical data of the insoluble fraction of aerosols collected at a land-based coastal station in SE Sardinia, Western Mediterranean ( $9^{\circ}$ long E, $39^{\circ}$ lat N) (Fig. 1) are presented, together with 
measurements on mineral particulate deposited with precipitation. A total of 86 samples (55 aerosol; 31 rain) were collected during the period October 1990/October 1991, and 69 of these (55 aerosol; 14 rain) are presented here. The remaining 17 rain samples were not analysed due to the low concentration of the insoluble fraction $\left(<2 \mathrm{mg} \mathrm{l}^{-1}\right)$.

In particular, aerosol sampling covered the period 1/10/1990-30/4/1991; precipitation was collected continuously during the period 1/10/1990-30/09/1991.

Two-to-three-day aerosol sampling (dry) and event-by-event rain collection (wet) were performed concurrently. Aerosol samples were collected with a high-volume $\left(60 \mathrm{~m}^{3} \mathrm{~h}^{-1}\right)$ Sierra Andersen ${ }^{\odot}$ sampler, using filters made of polyester as well as Whatman 41 filters. Sampling time was approximately 72 hours (air volume sampled $\sim 4000 \mathrm{~m}^{3}$ per sample).

Rain samples were collected using a Wet \& Dry (ARS 200 MTX $^{\odot}$ ) automatic collector.

\begin{abstract}
ANALYTICAL METHODS
The filters used for aerosol sampling are washed in acidified DDW, dried overnight and weighed before sampling. After collection, the filters were left overnight in the same dry-box and reweighed (to obtain the total suspended particulate value). This ensures that all samples are brought to the same degree of dryness, thus avoiding weighing errors. Then the exposed Hi-Vol filters were sonicated in a Milli-Q DD water bath for 20-30 minutes to dissolve salts and to resuspend insoluble particles from the filter. Solutions were filtered (on pre-weighted $0.22 \mu \mathrm{m}$ diameter Nuclepore ${ }^{\circledR}$ polycarbonate filters). Particles on the filters were dried in a dry-box and reweighed (to obtain the mineral suspended particulate value).

Rain samples were filtered through a pre-weighed $0.22 \mu \mathrm{m}$ diameter Nuclepore ${ }^{\odot}$ polycarbonate filter within a few hours of collection, and subdivided into two fractions, soluble and insoluble. The insoluble fraction was dried in a dry-box and re-weighed (to obtain the total particulate concentration value).

Major elements (Al, $\mathrm{Ca}, \mathrm{Fe}, \mathrm{K}, \mathrm{Mg}, \mathrm{Na}, \mathrm{Si})$ were analysed by atomic absorption spectrophotometry in insoluble aerosol particles and particles in precipitation. Trace elements $(\mathrm{Cd}, \mathrm{Cr}, \mathrm{Pb}, \mathrm{Zn})$ in the same samples were analysed in a graphite furnace.

Sampling method, sample treatment and analytical methods are described in detail in Guerzoni et al. (1993; 1997) and Molinaroli et al. (1993).

Back-trajectories from the sampling point were computed from analysed wind data along constant pressure surfaces, to help identify the potential source areas of the dust load. Trajectories were provided by NOAA (Harris, 1982).
\end{abstract}

\title{
STATISTICAL STRATEGY
}

The following steps summarise the strategy employed in the first study:

1. data were ranked to obtain a comparable unit of the variables, for representation of outliers and because the data were closed;

2. cluster analysis in $q$-mode (results cluster objects on the basis of values of variables) was performed using Ward's hierarchical agglomerative method and the squared Euclidean distance measure;

3. on the basis of the results of clustering, a number of sample groups was derived by applying a similarity level;

4. with this a priori criterion, linear discriminant analysis was employed to characterise sample groups in relation to variables with the greatest discriminating power.

In the second study: 
1. data were ranked as previously described;

2. factor analysis was used to reduce a large number of variables to a few uncorrelated variables (factors);

3. cluster analysis in q-mode was performed on significant factors (Ward's hierarchical agglomerative method and the squared Euclidean distance measure);

4. on the basis of the results of clustering linear discriminant analysis was employed.

This computational and statistical strategy allowed us to proceed gradually from simple data analysis to more sophisticated pattern recognition. In particular, all calculations in the analytical and multivariate statistics were based only on ranked data: each value was replaced by a number giving its place in the sequence from highest to lowest or vice versa (Swan and Sandilands, 1995). Ranked data allows application of methods with fewer assumptions about the underlying distribution (Lebart et al. 1984; Korhonen and Siljamäki, 1998). Rank transformation is also a suitable method for representing outliers and whenever standardisation is needed.

\section{RESULTS AND DISCUSSION}

\section{Measurements in air and rain}

Table 1 lists arithmetic and geometric means for insoluble fractions in aerosol and rain. The overall means are $6 \mu \mathrm{g} \mathrm{m}^{-3}$ and $26 \mathrm{mg} \mathrm{l}^{-1}$, with significant differences between samples collected from winds from the south, called Saharan, and samples from all other directions, called Background. This classification was also carried out prior to chemical analysis, using a suite of meteorological data, together with backtrajectories. Table 2 shows the wind component of particle concentrations in Saharan and Background aerosols. It may be seen that maximum load concentrations are related to S-SE and NW winds. Other authors have shown that southern winds in the Mediterranean carry great quantities of desert-derived particles (Chester et al., 1993b; Loÿe-Pilot and Martin, 1996). Dust transport across the northern boundaries of the Sahara into the Western Mediterranean and Europe is approximately $12 \%$ of the total, i.e. 100 million tons. These estimates are subject to considerable uncertainties in relation to large-scale meteorological features (dry years, ITCZ latitudinal position) which give rise to strong interannual variability. Direct measurements (mainly bulk deposition; Loÿe-Pilot et al., 1995; Le Bolloch and Guerzoni, 1995) and historical inventories of occurrence of dust rain or dust haze in Europe (e.g. Bücher, 1989) indicate maximum fallout in spring and autumn.

\section{Chemical data}

Table 3 lists averages and geometric means of the insoluble fraction in aerosols and precipitation. The content of particulate concentration in rain is similar to that of aerosols for the major elements, and frequently lower for trace elements. The distributions of most elements are log-normal. These data have already been compared with other data from coastal areas in the Mediterranean (Corsica, Blanes, Tour du Valat, Cap Ferrat) (Guerzoni et al., 1996). The result is an increase in trace metal concentrations westwards along Mediterranean coastal areas. 


\section{First statistical treatment}

Treatment was applied to the chemical data of the insoluble fraction, from aerosols and precipitation together. CA was applied to the ranked data, and results are shown in Fig. 2. Objects grouped together on numerical grounds form a cluster of points in the multivariate space spanned by the elements. Five groups emerge using the similarity value (Dlink/Dmax cut-off of 5). The main result of this kind of representation is that, based on their similarity coefficient, all samples fall in five groups, identifying aerosol depositional mode. This classification distinguishes rain samples (groups 2 and, to a certain extent, 5) from others, but mixes Saharan samples with other Local aerosols (group 1) and Mediterranean Background samples with ones from Local mining areas (groups 3 and 4).

DA was applied to the 5 groups plotting in the space of the first two discriminant functions (Fig. 3). DA is used to distinguish statistically between two or more predefined groups of samples on the basis of multiple variables. It contains tests for establishing the rate of success for discriminating variables when they are combined into a discriminating function, and also criteria for controlling stepwise selection of variables according to their discriminating power. DA proceeds by calculating linear discriminant functions: linear combinations of the original variables which maximise the differences between the groups

$$
\mathrm{F}_{\mathrm{k}}=\mathrm{a}_{1} \mathrm{~V}_{1}+\mathrm{a}_{2} \mathrm{~V}_{2} \ldots \ldots \ldots
$$

where $V_{1}$ are the elements and $a_{1}$ are the discriminant coefficients, the value of $F$ for an object is called its discriminant score. These computations are described by Nie et al. (1975) and Rock (1988).

The elements with greater discriminating power were: (a) $\mathrm{Pb}$ for function 1, separating groups 2 (rain) from 3 and 4 (Background pollution and Local mining areas); (b) $\mathrm{Al}$ and $\mathrm{Fe}$ for function 2, separating groups 1 (Saharan and Local) from 5 (Rain and mixed aerosol). Inspection of the various aerosol groups with independent characteristics, i.e., grain-size, mineralogy, wind speed and direction, confirmed the unsatisfactory grouping of this classification.

\section{Second statistical treatment}

The second treatment was applied to the same ranked data set, using Q-FA as a first step. Q-Factor analysis transforms the data matrix using cos theta as similarity method, generating a matrix of $\mathrm{m} \times \mathrm{m}$ objects data. This technique applied to our data set was also useful in focusing better on the principal assemblages as source markers. Table 4 shows the factor score matrix, with indications of the percentage of information explained by each factor. It may be seen that approximately $10 \%$ of the information is lost in the next few steps. Three factors explain $90.5 \%$ of the variability and the first two factors represent $72.4 \%$ of total variability. Factor 1 (36.4\%) represents crust-enriched aerosols, partly regional background and mostly Saharan dusts. Factor 2 is mainly representative of Background anthropogenic aerosols, mixed with $\mathrm{Pb}$-enriched mining tails. Factor 3 typically has the signature of Local mining sources. Since some of the trace elements are common to Factors 2 and $3(\mathrm{~Pb}, \mathrm{Cd}$ and $\mathrm{Zn}$ in particular), Q-FA was not able to distinguish Local from distant sources. Back-trajectories (Harris, 1982) of samples closest to each group are shown in Fig. 4 and were used to trace possible sources.

The next step was to apply CA to the factor loading matrix. Results are shown in Fig 5. The procedure revealed the "highest" variability part of the data set, omitting the less 
significant part (which may be defined as "background noise" and was therefore not particularly significant for the data set). In this case too, five groups were extracted by applying the same cut-off level. This classification was able to distinguish: (i) crustenriched aerosols, due to regional background plus Saharan dusts (group 2, corresponding to Factor 1 of Q-FA); (ii) samples of Background anthropogenic aerosols, mixed with $\mathrm{Pb}$ enriched mining tails (groups 3 and 4, corresponding to Factor 2). Instead, CA combined samples with the signature of Local mining sources (Factor 3) with other Local aerosols and Mediterranean Background (groups 5 and 1).

The five groups of samples obtained by clustering were used to perform DA. Results are shown in Fig. 6, in which discriminant scores are scatter-plotted for the two discriminant functions. Elements with greater discriminating power are: (i) $\mathrm{Pb}, \mathrm{Cd}$ and $\mathrm{Zn}$ for function 1, separating especially group 2 (Saharan and rain) from 3 (Local mining areas), slightly from 4 (Background pollution), 5 (Local) and 1 (Mixed); (ii) $\mathrm{Na}$ for function 2, which clearly separates group 4 (Background pollution) from 5 (Local) and slightly from all the others. All discriminating elements therefore tend to discern Sardinian contributions from long-range ones.

In this case too, the significance of the five groups was checked with independent information, i.e., geological signature. In particular, Saharan dust was separated from other aerosols and put together with rain, most of them crust-enriched. Local inputs were included in group 3, from mining areas (old sphalerite and galena mining tailings, located about 100-200 km from the sampling site) and group 5 (Local outcrops). Group 4 represented long-range Background air pollution associated with N-NW trajectories. Group 1 contained mixed aerosols, in which the long sampling time (72 hours) favoured a complex mixture from different sources. Granulometric, mineralogical and meteorological data supported this interpretation. Grain-size analysis highlighted the well-known bimodality distribution of Saharan aerosols, combined with the typical mineralogical markers of desert provenance (i.e., palygorskite, kaolinite, rounded quartz, calcite) as well as wind direction (E-SE) and the highest mass loadings. As an example, Fig. 7 shows some cumulative volume grain-size distributions of Saharan aerosol events, two different episodes (in autumn and spring) showing distinct grain-size distributions (Molinaroli and De Falco, 1995). Samples were also examined by scanning electron microscopy (SEM); Fig. 8 shows three SEM photographs of typical mineral markers observed in Saharan and Local aerosols.

Wind speed (high: $15-18 \mathrm{~m} / \mathrm{s}$ ) and wind direction (west), together with very fine grain sizes $(1-2 \mu \mathrm{m})$ and mineralogical composition (talc, amphibole, Na-plagioclase, etc.) supported Local and regional inputs for groups 3 and 5.

\section{CONCLUSIONS}

Concurrent sampling of aerosol and precipitation is useful in comparing contents of the insoluble fraction of air particulate.

The statistical methods used in this study are well known, and commonly used in situations in which the interplay of many sources of variation do not allow an objective and comprehensive method of describing physical phenomena. Divergent results are obtained by applying two different combinations of classification and statistical methods to geochemical data. 
In the first statistical treatment, aerosols and precipitation particles were better distinguished, but little information could be inferred for separating Local from distant sources.

In the second statistical treatment, the application of CA subsequent to Q-FA better distinguished among Saharan dust, remote Background pollution and Local aerosol sources, but some unexplained mixed aerosols still resulted. Subsequent DA better discriminated Sardinian contributions from long-range ones; moreover, crust-enriched precipitation was classified together with Saharan dust, indicating that they are similar to desert-derived aerosols.

Granulometric, mineralogical and meteorological data confirmed the results of the statistical methods employed in the second treatment.

\section{ACKNOWLEDGEMENTS}

This paper is IGM-CNR No. 1083. We thank F. Visin and G. Quarantotto for chemistry work, and G. Walton for revision of the English text. We also thank Prof. P.Mantovan and Dr. A. Pastore for assistance in the review of the statistical procedures 


\section{REFERENCES}

Bergametti G., Gomes L., Remoudaki E., Desbois M., Martin D. and Buat-Menard P. (1989) Present-day transport and deposition patterns of African dusts to the NorthWestern Mediterranean. In Paleoclimatology and Paleometeorology: Modern and Past Patterns of Global Atmospheric Transport (eds M. Leinen and M. Sarnthein), pp. 227252. Kluwer Academic Publishers, Dordrecht.

Bücher A. (1989) Fallout of Saharan dust in the northern Mediterranean region. In Paleoclimatology and Paleometeorology: Modern and Past Patterns of Global Atmospheric Transport (eds M. Leinen and M. Sarnthein), pp. 565-584. Kluwer Academic Publisher, Dordrecht.

Chester R., Nimmo M., Alarcon M., Saydam C., Murphy K.J.T., Sanders G.S. and Corcoran P. (1993a) Defining the chemical character of aerosols from the atmosphere of the Mediterranean Sea and surrounding regions. Oceanol. Acta 16 (3), 231-245.

Chester R., Murphy K.J.T., Lin F.J., Berry A.S., Bradshaw B.A. and Corcoran P. (1993b) Factors controlling the solubilities of trace metals from non-remote aerosols deposited to the sea surface by the "dry" deposition mode. Marine Chemistry 42, 107-126.

Correggiari A., Guerzoni S., Lenaz R., Quarantotto G. and Rampazzo G. (1989) Dust deposition in the Central Mediterranean (Tyrrhenian and Adriatic Seas): relationship with marine sediments and riverine input. Terra Nova 1, 549-558.

GESAMP (1989) The atmospheric input of trace species to the world oceans. Reports and Studies 38, $111 \mathrm{p}$.

Guerzoni S., Landuzzi W., Lenaz R., Quarantotto G., Rampazzo G., Molinaroli E., Turetta C., Visin F., Cesari G. and Cristini S. (1993) Fluxes of soluble and insoluble metals and nutrients from the atmosphere to the Central Mediterranean Sea. Water Poll. Reas. Rep. 30, 438-493.

Guerzoni S., Quarantotto G., Cesari G., Molinaroli E., Rampazzo G. and Le Bolloch O. (1996) Trace metal composition and grain-size of particulates in aerosols and precipitation collected in N.W. Mediterranean $\left(39^{\circ} \mathrm{N}, 9^{\circ} \mathrm{E}\right)$ : a multivariate analyses. In The Impact of Desert Dust Across the Mediterranean (eds S. Guerzoni and R. Chester), pp. 333-338. Kluwer Academic Publishers, Dordrecht.

Guerzoni S., Molinaroli E. and Chester R. (1997) Saharan dust inputs to the W. Mediterranean Sea: depositional patterns, geochemistry and sedimentological implications. Deep-Sea Res. II 44 (3-4), 631-654.

Harris J.M. (1982) The GMCC atmospheric trajectories program. In NOAA Technical memorandum En ARL-116, pp. 32. NOAA, Boulder, Colorado.

Hopke P.K. (1988) Target transformation factor analysis as an aerosol mass apportionment method: a review and sensitivity study. Atmos. Environ. 22 (9), 1777-1792.

Korhonen P. and Siljamäki A. (1998) Ordinal principal component analysis. Theory and application. Computational Statistics \& Data Analysis 26, 411-424.

Lebart L., Morineau A. and Warwick K.M. (1984) Multivariate descriptive statistical analysis. Wiley and Sons, New York.

Le Bolloch O. and Guerzoni S. (1995) Acid and alkaline deposition in precipitation on the Western coast of Sardinia, Central Mediterranean $\left(40^{\circ} \mathrm{N}, 8^{\circ} \mathrm{E}\right)$. Water Air and Soil Pollution 85 (4), 2155-2160.

Loÿe-Pilot M.D. and Martin J.M. (1996) Saharan dust input to the Western Mediterranean: an eleven years record in Corsica. In The Impact of Desert Dust Across the 
Mediterranean (eds S. Guerzoni and R. Chester), pp. 191-199. Kluwer Academic Publishers, Dordrecht.

Molinaroli E., Guerzoni S. and Rampazzo G. (1993) Contribution of Saharan dust to the Central Mediterranean Basin. In Processes Controlling the Composition of Clastic Sediments (eds M. Johnsson and A. Basu), SP 284, pp. 303-312. Geol. Soc. America.

Molinaroli E. and De Falco G. (1995) Grain size analysis of atmospheric particulates in aerosol and rain collected in Sardinia (Italy). Giornale di Geologia 57 (1-2), 59-65.

Morandi M. T., Daisey J.M. and Lioy P.J. (1987) Development of a modified factor analysis/multiple regression model to apportion suspended particulate matter in a complex urban airshed. Atmos. Environ. 21 (8), 1821-1831.

Nie H.H., Hull C.H., Jenkins J.G., Steinbrenner K. and Bent D.H. (1975) SPSS: statistical package for the social sciences. McGraw-Hill Book Co., New York.

Prospero J.M. (1996) Saharan dust transport over the North Atlantic Ocean and Mediterranean: an overview. In The Impact of Desert Dust Across the Mediterranean (eds S. Guerzoni and R. Chester), pp. 133-151. Kluwer Academic Publishers, Dordrecht.

Rock N.M.S. (1988) Numerical Geology. Springer-Verlag.

Swan A.R.H. and Sandilands M. (1995) Introduction to geological data analysis. Science, Oxford.

Van Malderen H., Rojas C. and Van Grleken R. (1992) Characterization of individual giant aerosol particles above the North Sea. Environ. Sci. Technol. 26, 750-758. 


\section{FIGURE CAPTIONS}

Fig. 1 Mediterranean basin and location of sampling station.

Fig. 2 Dendrogram produced by clustering 69 samples. Cluster analysis was performed on ranked data matrix using Ward's hierarchical agglomerative method and squared Euclidean distance measure. Numbers (1-5) indicate clusters identified by the classification.

Fig. 3 Discriminant score scatterplot for two discriminant functions: classification of five groups of samples obtained by cluster analysis (see Fig. 2). Discriminant variables are: $\mathrm{Pb}$ for Function $1 ; \mathrm{Al}$ and $\mathrm{Fe}$ for Function 2.

Fig. 4 Back-trajectories computed at $850 \mathrm{hPa}$ level. Symbols mark every 12 hours along wind path.

Fig. 5 Dendrogram produced by clustering 69 samples. Cluster analysis was performed on Q-FA scores using Ward's hierarchical agglomerative method and squared Euclidean distance measure.

Fig. 6 Discriminant score scatterplot for three discriminant functions: classification of sample groups obtained by cluster analysis (see Fig. 5). Discriminant variables are $\mathrm{Pb}, \mathrm{Cd}, \mathrm{Zn}$ for Function 1; Na for Function 2.

Fig. 7 Cumulative volume grain-size distribution of Saharan dust transported over Sardinia during autumn 1990 (solid line) and apring 1991 (broken dashed line).

Fig. 8 Scanning electron micrographs of marker minerals. (a) Perfectly rounded palygorskite grain with felt-like surface texture, of desert type. (b) Talc grain typical of Local mining area. (c) Na-rich plagioclase grain typical of Local outcrops. 


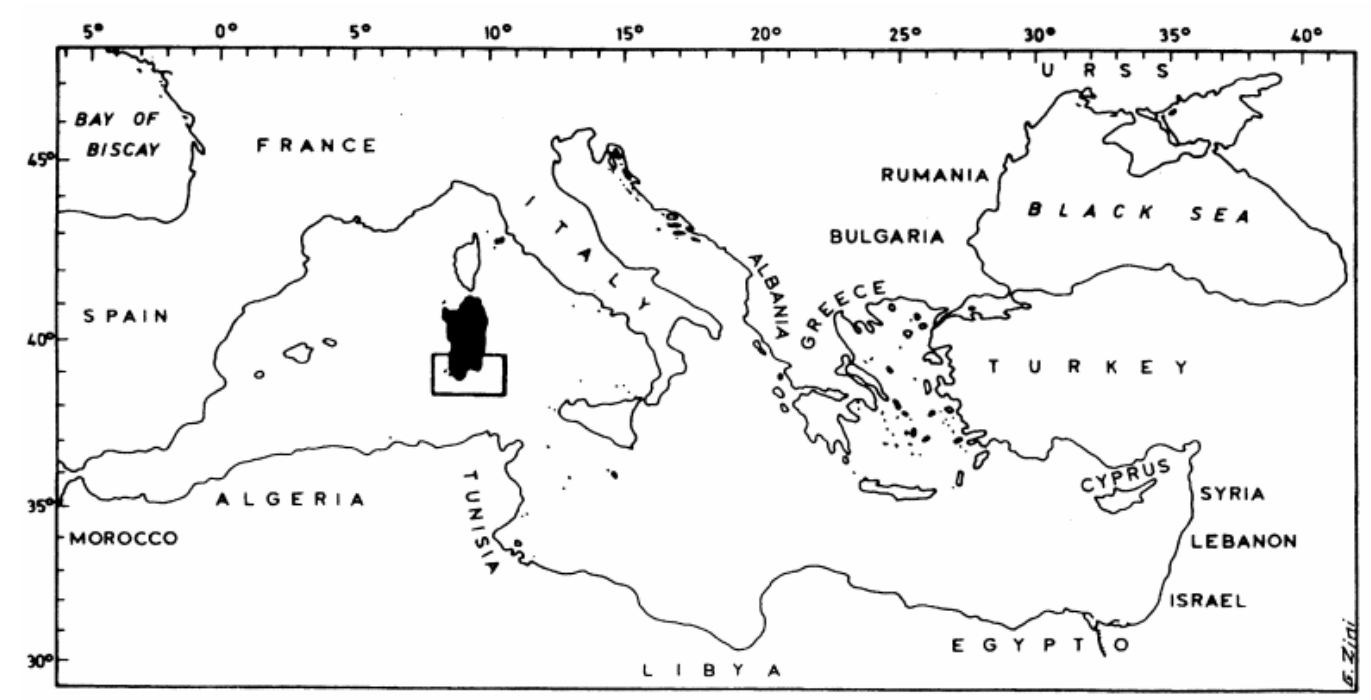

FIG. 1

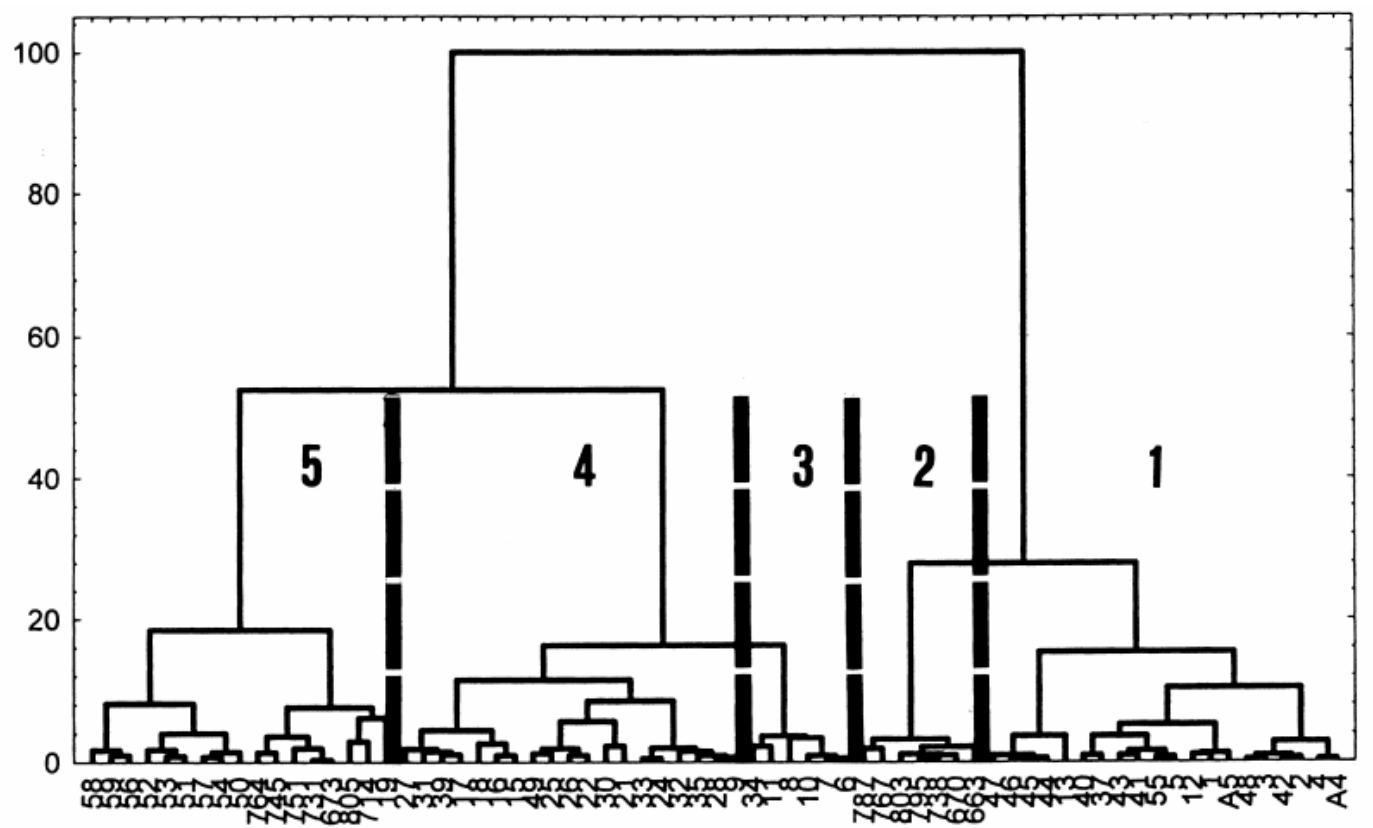

FIG. 2 


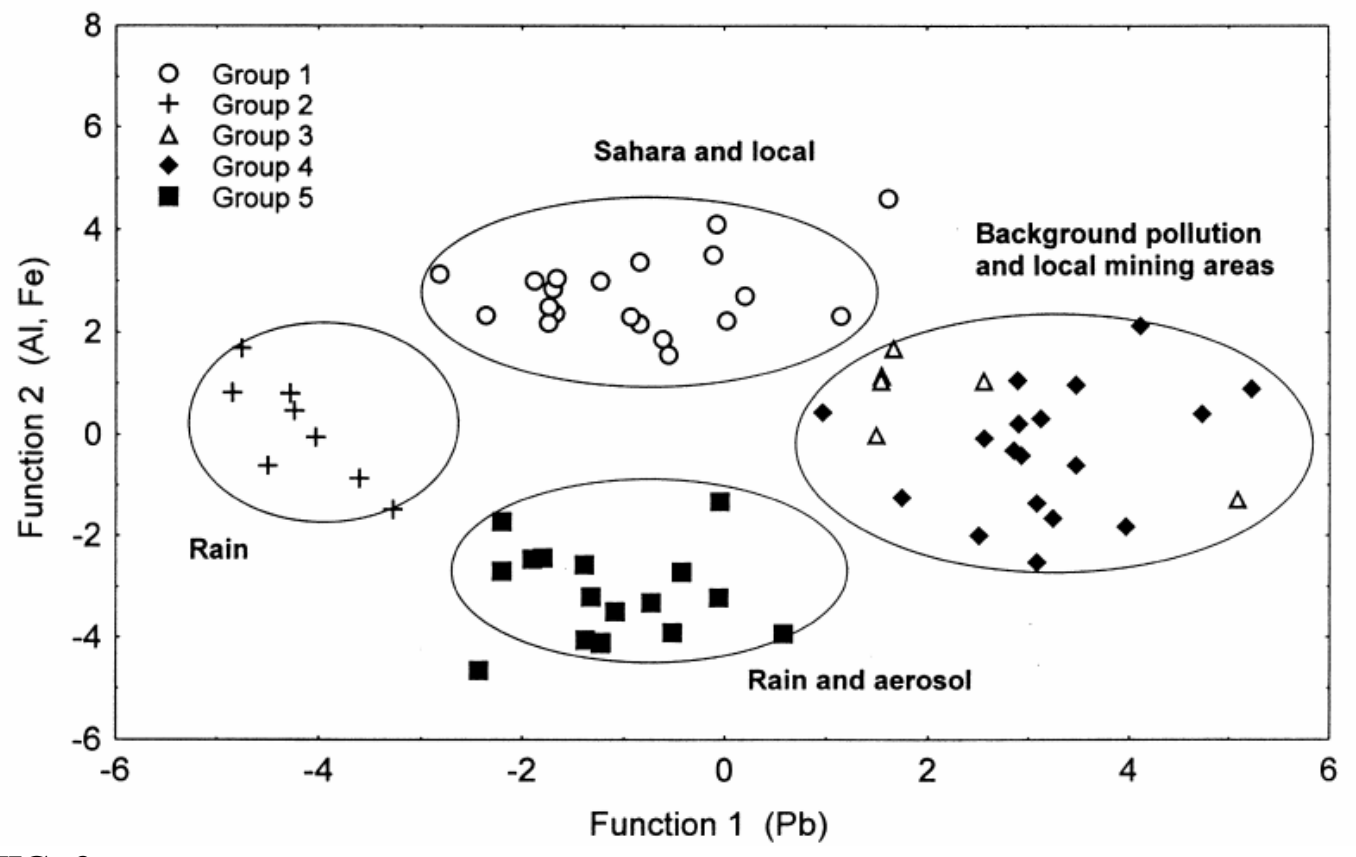

FIG. 3

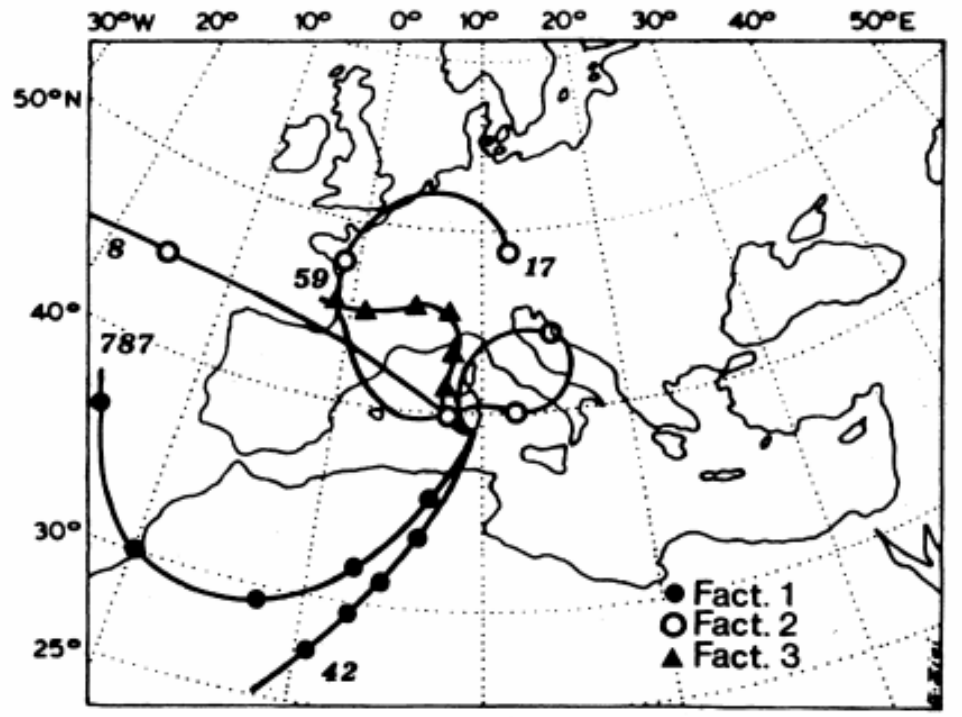

FIG. 4 


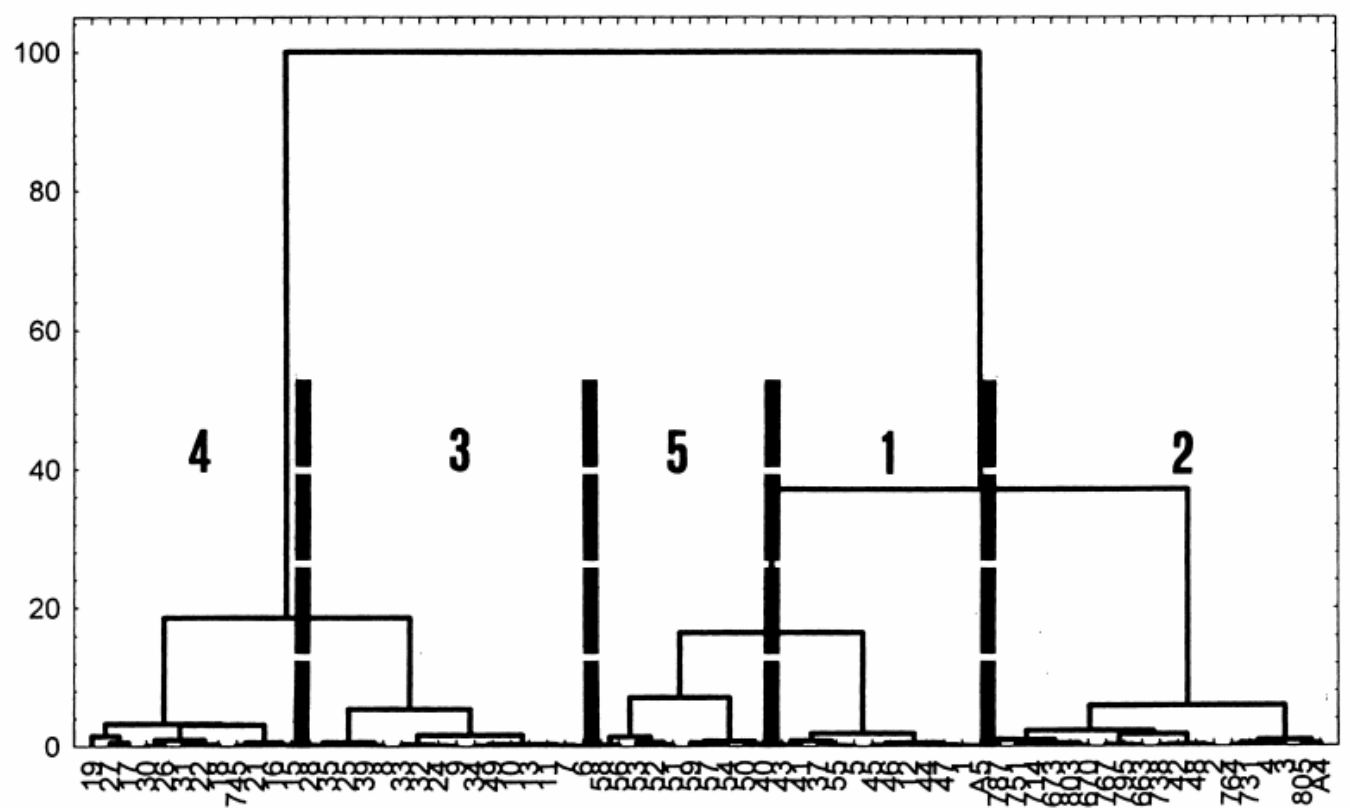

FIG. 5

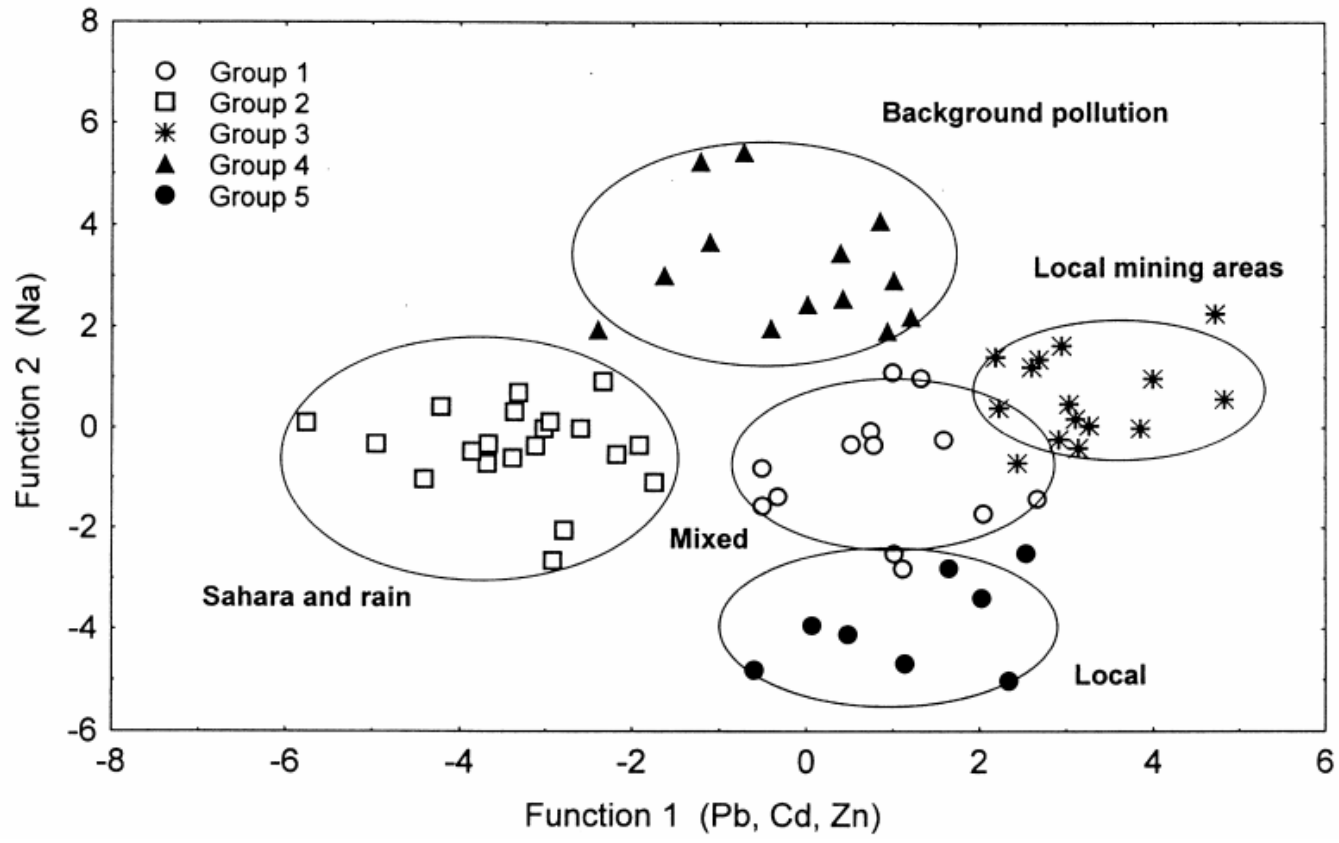

FIG. 6 


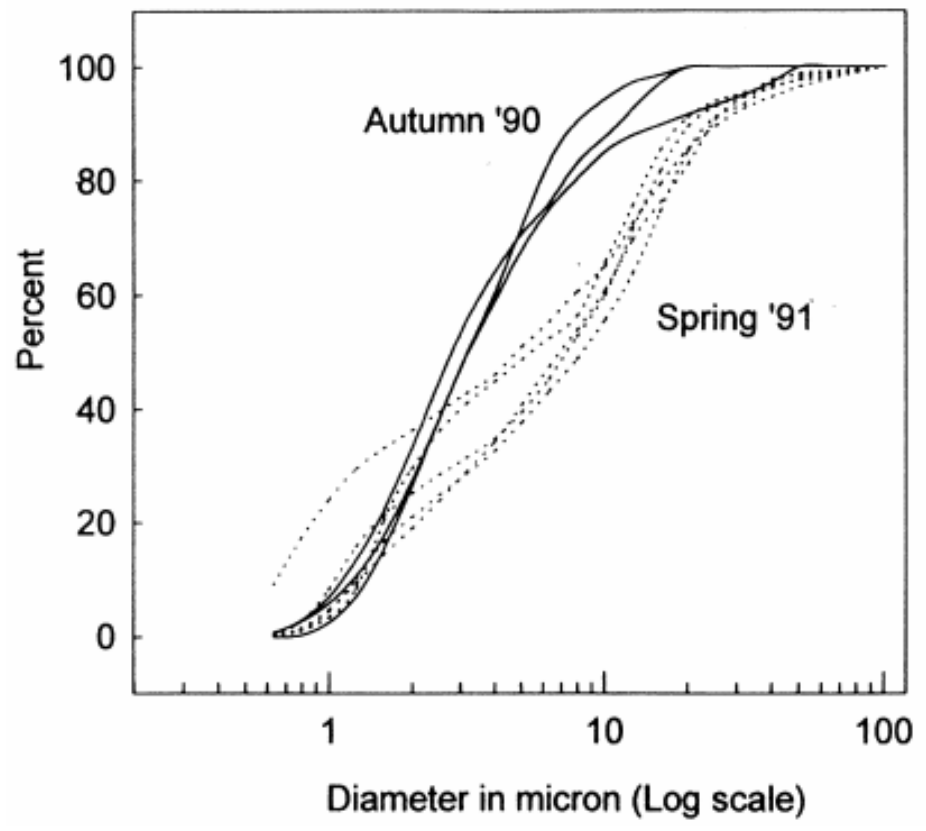

FIG. 7 

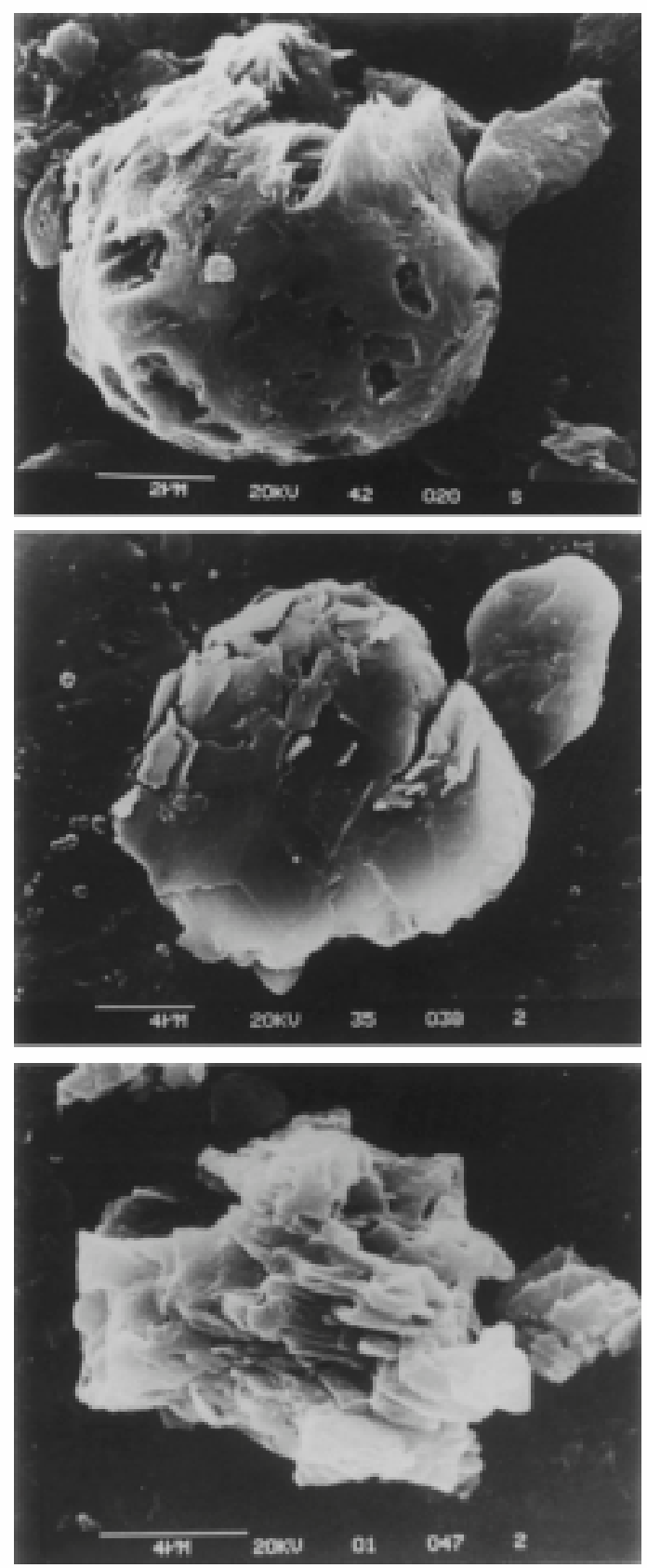

FIG. 8 
Table 1. Insoluble fractions in Saharan and Background aerosol and precipitation. Arithmetic mean (Geometric mean).

\begin{tabular}{lccc}
\hline & Saharan & Background & All \\
\hline Aerosol & $(\mathbf{n = 1 0})$ & $(\mathbf{n}=\mathbf{4 5})$ & $(\mathbf{n}=\mathbf{5 5})$ \\
$\left(\mu \mathrm{g} \mathrm{m}^{-3}\right)$ & $21(17)$ & $3(2)$ & $6(4)$ \\
Rain $_{\left(\mathrm{mg} \mathrm{l}^{-1}\right)}$ & $(\mathbf{n = 1 2})$ & $(\mathbf{n = 1 9})$ & $(\mathbf{n = 3 1})$ \\
\hline
\end{tabular}

Table 2. Wind component of particle concentration in air for Saharan and Background aerosols.

\begin{tabular}{ccc}
\hline $\begin{array}{c}\text { Wind } \\
\text { direction }\end{array}$ & $\begin{array}{c}\text { Saharan } \\
(\mathbf{n = 1 0}) \\
\mu \mathrm{g} \mathrm{m}^{-3}\end{array}$ & $\begin{array}{c}\text { Background } \\
(\mathbf{n = 4 5}) \\
\mu \mathrm{g} \mathrm{m}^{-3}\end{array}$ \\
\hline $\mathrm{NE}$ & 0.1 & 0.1 \\
$\mathrm{E}$ & 3.2 & 0.4 \\
$\mathrm{SE}$ & 10.0 & 0.5 \\
$\mathrm{~S}$ & 2.3 & 0.1 \\
$\mathrm{SW}$ & 1.7 & 0.1 \\
$\mathrm{~W}$ & 1.4 & 0.3 \\
$\mathrm{NW}$ & 2.2 & 1.4 \\
$\mathrm{~N}$ & 0.1 & 0.2 \\
Total & $\mathbf{2 1 . 0}$ & $\mathbf{3 . 1}$ \\
\hline
\end{tabular}

Table 3. Chemical data of insoluble fraction of aerosols and precipitation. Averages with geometric means in brackets.

\begin{tabular}{lccc}
\hline Element & $\begin{array}{c}\text { Aerosol } \\
(\%)\end{array}$ & $\begin{array}{c}\text { Rain } \\
(\%)\end{array}$ & $\begin{array}{c}\text { Aerosol } \\
\left(\mu \mathrm{g} \mathrm{m}^{-3}\right)\end{array}$ \\
\hline $\mathrm{Si}$ & $16.5(14.0)$ & $17.5(15.6)$ & $1.182(0.459)$ \\
$\mathrm{Al}$ & $5.5(4.4)$ & $5.2(3.9)$ & $0.526(0.143)$ \\
$\mathrm{Ca}$ & $0.87(0.51)$ & $1.1(0.7)$ & $0.110(0.017)$ \\
$\mathrm{Na}$ & $5.0(2.4)$ & $2.0(0.9)$ & $0.241(0.080)$ \\
$\mathrm{K}$ & $2.1(1.0)$ & $1.8(1.5)$ & $0.095(0.034)$ \\
$\mathrm{Mg}$ & $1.0(0.8)$ & $1.3(1.1)$. & $0.092(0.026)$ \\
$\mathrm{Fe}$ & $2.9(2.6)$ & $3.1(2.8)$ & $0.228(0.085)$ \\
\hline & $\left(\mathrm{mg} \mathrm{kg}^{-1}\right)$ & $\left(\mathrm{mg} \mathrm{kg}^{-1}\right)$ & $\left(\mu \mathrm{g} \mathrm{m}^{-3}\right)$ \\
\hline $\mathrm{Cd}$ & $6.5(4.4)$ & $1.4(1.2)$ & $0.000026(0.016)$ \\
$\mathrm{Zn}$ & $1216(1045)$ & $552(444)$ & $0.0066(0.0034)$ \\
$\mathrm{Pb}$ & $3324(2213)$ & $191(153)$ & $0.0112(0.0073)$ \\
$\mathrm{Cr}$ & $82(65)$ & $32(27)$ & $0.0006(0.0003)$ \\
\hline
\end{tabular}


Table 4. Q-Mode Factor score matrix. Elements with asterisks are those characterising each factor.

\begin{tabular}{cccc}
\hline Variable & Fact. 1 & Fact. 2 & Fact. 3 \\
& & & \\
\hline $\mathrm{Si}$ & $1.097^{*}$ & 0.44 & 0.00 \\
$\mathrm{Al}$ & $1.145^{*}$ & 0.11 & 0.41 \\
$\mathrm{Ca}$ & $1.162^{*}$ & 0.46 & -0.08 \\
$\mathrm{Na}$ & 0.34 & $2.077^{*}$ & -1.15 \\
$\mathrm{~K}$ & $1.626^{*}$ & -0.39 & 0.51 \\
$\mathrm{Mg}$ & $1.591^{*}$ & -0.31 & 0.42 \\
$\mathrm{Fe}$ & $1.118^{*}$ & 0.20 & 0.32 \\
$\mathrm{Cd}$ & -0.41 & 0.75 & $1.807^{*}$ \\
$\mathrm{Zn}$ & -0.33 & 0.32 & $2.303^{*}$ \\
$\mathrm{~Pb}$ & -0.52 & $1.740^{*}$ & 0.56 \\
$\mathrm{Cr}$ & 0.24 & $1.514^{*}$ & -0.28 \\
\hline \% of variance explained by each factor \\
\multicolumn{4}{c}{ Cumulative \% variance } \\
\multicolumn{4}{c}{36.0} \\
\hline
\end{tabular}

